\title{
Schizophrenia: what's DAO and DAOA got to do with it?
}

\begin{abstract}
Schizophrenia is a complex disease with a high heritability rate of about $80 \%$. Many linkage and association analysis revealed different chromosomal regions as candidates and functional studies showed the gene expression differences for many genes. Among them there are two interacting proteins found on the candidate regionsD-amino oxidase (DAO) and D-amino oxidase activator (DAOA). DAO oxidizes $\mathrm{D}$-amino acids notably $\mathrm{D}$-serine in the brain. $\mathrm{D}$-serine is a coagonist of the endogenous $\mathrm{N}$-methyl D-aspartate receptor (NMDAR). The hypofunction of N-methyl-D-aspartate glutamatergic neurotransmission has an important effect in the pathophysiology of the disease. DAOA is shown to be activating DAO. Because of their functions these two proteins are thought to be related with schizophrenia. Association studies also showed that these two are associated with schizophrenia in some populations from different regions of the world. Although positive associations were shown in these populations, in the others researchers failed to replicate that data. It is a common concept for schizophrenia association studies to have conflicting results in many candidate genes for different populations. Here we try to summarize the studies that were targeting the effect of DAO and DAOA SNPs in schizophrenia.
\end{abstract}

Volume I Issue 3 - 2014

\author{
Ceren Acar,' Sukru Kartalci² \\ 'Molecular Biology and Genetics Department, Inonu University, \\ Turkey \\ ${ }^{2}$ Psychiatry Department, Inonu University, Turkey
}

Correspondence: Ceren Acar, Molecular Biology and Genetics Department, Inonu University, 44280, Malatya, Turkey, Tel 04223773817,Email ceren.acar@inonu.edu.tr

Received: July 14, 2014 | Published: July 18, 2014

Keywords: DAO, DAOA, G72, schizophrenia, SNP, population study

Abbreviations: GWAS, genome-wide association studies; DAO, d-amino oxidase; DAOA, d-amino oxidase activator; SNP, single nucleotide polymorphism; NMDAR, n-methyl d-aspartate receptor

\section{Introduction}

Schizophrenia is a common chronic mental disorder which affects $\sim 1 \%$ of the world's population. ${ }^{1}$ It is a major cause of morbidity and patients need long term medical and social care. It is caused by both environmental and genetic factors. The contribution of inheritance to the disease is $\sim 80 \%$ and family, twin, and adoption studies showed the importance of genes on the susceptibility to disease. Genome-wide association analysis (GWAS), case-control studies, and meta-analysis also demonstrate the genetic risks of the disease. ${ }^{2}$ From the genetic epidemiologic studies it is known that the mode of transmission of schizophrenia is complex.

Several linkage studies were performed to point out the candidate regions for schizophrenia. These studies have found linkage for different localizations of the genome at the significant level. There are many association studies with these candidate regions..$^{3-10}$ But the results of the positive or negative associations should be replicated by population studies with large number of individuals. One of the candidates that was found in these studies was DAOA(D-amino oxidase activator) gene. In 2002, Chumakov and colleagues published a paper about the association to a new human gene- G72. ${ }^{11}$ They focused on 13q22-q34 chromosomal region and by doing single point association analysis, population analyis with different groups and gene discovery and functional studies, they figured out the interaction in between the G72 and DAO genes. Yeast two hybrid experiments performed with G72 protein identified G72 as an interacting partner with D-amino oxidase (DAO) enzyme. In vitro studies revealed that DAO enzyme activity was enhanced with G72. Hence it has been named D-amino acidoxidase activator (DAOA). The interaction of these two genes points the involvement of $\mathrm{N}$-methyl $\mathrm{D}$ - aspartate receptor regulation pathway in schizophrenia. Because of their functions in the brain and their chromosomal localizations these two interacting partners are candidates for the studies about the molecular mechanisms underlying schizophrenia. In this study we try to summarize the literature on schizophrenia relation with DAO and DAOA genes.

\section{DAO and DAOA}

DAO gene is located at chromosome $12 \mathrm{q} 24$, it has 11 exons and full length transcript of $1595 \mathrm{bp}$. Iten codes $39 \mathrm{kDa}$ flavoenzyme D-amino oxidasewith 347 amino acids. Itoxidizes D-amino acids notably $\mathrm{D}$-serine which is a coagonist of the endogenous N-methyl D-aspartate receptor (NMDAR). DAOA gene is located at chromosome 13q34 andthe gene spans $29 \mathrm{~kb}$. G72 and G30 overlaps on the complementary strands of the chromosome and they are transcribed in the opposite direction. ${ }^{12}$

Glutamatergic neurotransmission abnormalities were shown to be involved in schizophrenia pathogenesis. The hypofunction of N-methyl-D-aspartate glutamatergic neurotransmission has an important effect in the pathophysiology of the disease. ${ }^{13}$ Several studies showed that D-serine facilitates the NMDAR function and DAO activity is increased in the case of schizophrenia. ${ }^{14} \mathrm{D}$-serine is a coagonist for NMDAR and DAO gene product-D-amino acid oxidase enzyme- degrades D-serine. On the other hand DAOA gene product D-amino acid oxidase activator-activates DAO enzyme. ${ }^{15}$ Because of their role in NMDAR signalling and by being interaction partners DAO and DAOA studies were good examples for investigating the molecular complexity of schizophrenia.

\section{Population studies}

Several association studies with schizophrenia patients from 
different ethnic backgrounds were performed by different research groups. PubMed search of "schizophrenia and DAO" revealed 71 articles. These include both mechanistic work and association studies together when we narrow the search with the keywords (DAO SNPs and schizophrenia there are 22 articles are found in between 2005 and 2013. There were 112 articles for "DAOA gene and schizophrenia" 63 of which were about DAOA SNPs and schizophrenia. But the association studies with different populations displayed conflicting results. Many groups reported significant associations with DAOA gene at the allelic, genotypic and haplotypic level for the different populations and the sample size.

Chumakov's findings were the first study that showed the relation of DAOA and schizophrenia. They also showed the association between DAO SNPs and the disease. They reported the association of four SNPs with schizophrenia in Canadian samples. ${ }^{11}$ Schumacher et al. ${ }^{16}$ Reported the relationship of DAO and DAOA variations in German population. Yang et al., ${ }^{15}$ reported a significant association of DAO SNPs (DAO7-DAO8-DAO13) in Taiwan Han Chinese population.

On the other side there are reports about lack of association between polymorphisms of DAO and DAOA in different ethnic groups. As an example Bass et al., ${ }^{17}$ couldn't find an association in their patients from UK. Like Sacchetti et al., ${ }^{18}$ couldn't find an association with the SNPs of DAO and DAOA in their group of patients from Northern Italy. In our group of patients from Turkey we couldn't find an association as well (Acar and Kartalci, unpublished study). A study that was performed by Chung et al., ${ }^{19}$ with Korean schizophrenia patients investigated the relationship of aggressive behaviour in schizoprenia patients and they also couldn't find an association neither with DAO nor DAOA. By looking at these and manyothers, it is possible to think these results as false positives or false negatives depending on the sample size or SNP numbers that are involved in these studies. But onething should be kept in mind that there should be more mechanistic work and proteomic data to support or understand the effect of these two genes or the other schizophrenia candidate genes. Because it is known that DAO expression and enzyme activity is increased in the case of this disorder. ${ }^{20}$ Besides depending on the presence of positive or negative symptoms of the disease, different candidate genes may be taking part in the pathology of the disease.

\section{Conclusion}

Schizophrenia is a mental disorder affecting about 30 million people worldwide. It should be noted that schizophrenia is a very complex disease and it is not caused by only one mutation in a particular gene. The interaction of environmental factors and genetic architecture of the individuals both contribute to the disease pathology. Genetic architecture includes sequence variations, gene function and differential protein expression. When these factors are taken into account it is reasonable to combine genomics and proteomics to understand the molecular pathology of this global health problem. Searching for candidate genes, and association studies, can go hand in hand with post mortem brain studies, cell culture, and animal models in order to enlighten the molecular mechanisms underlying schizophrenia. By this way novel therapeutic targets or effective treatment methods can be found

\section{Acknowledgements}

Authors would like to thank M. MertSozen, $\mathrm{PhD}$ for his valuable comments on manuscript's preparation.

\section{Conflict of interest}

The author declares no conflict of interest.

\section{References}

1. Bromet EJ, Fenning S. Epidemiology and natural history of schizophrenia. Biol Psychiatry. 1999;46(7):871-881

2. Tan J, Lin Y, Su L, et al. Association between DAOA gene polymorphisms and the risk of schizophrenia, bipolar disorder and depressive disorder Prog Neuropsychopharmacol Biol Psychiatry. 2014;51:89-98.

3. Craddock N, O'Donovan MC, Owen MJ. The genetics of schizophrenia and bipolar disorder: dissecting psychosis. JMed Genet. 2005;42(3):193204.

4. Blouin JL, Dombroski BA, Nath SK, et al. Schizophrenia susceptibility loci on chromosomes 13q32 and 8p21. Nat Genet. 1998;20(1):70-73.

5. Brzustowicz LM, Honer WG, Chow EW, et al. Linkage of familial schizophrenia to chromosome 13q32. Am JHum Genet. 1999;65(4):10961103.

6. Camp NJ, Neuhausen SL, Tiobech J, et al. Genomewide multipoint linkage analysis of seven extended Palauan pedigrees with schizophrenia, by a Markov-chain Monte Carlo method. Am J Hum Genet. 2001;69(6):1278-1289.

7. Faraone SV, Skol AD, Tsuang DW, et al. Linkage of chromosome 13q32 to schizophrenia in a large veterans affairs cooperative study sample. $\mathrm{Am}$ J Med Genet. 2002;114(6):598-604.

8. Levinson DF, Holmans P, Straub RE, et al. Multicenter linkage study of schizophrenia candidate regionson chromosomes $5 \mathrm{q}, 6 \mathrm{q}, 10 \mathrm{p}$, and 13q:schizophrenia linkage collaborative group III. Am J Hum Genet. 2000;67(3):652-663.

9. Lin MW, Curtis D, Williams N, et al. Suggestive evidence linkage of schizophrenia to markers on chromosome 13q14.I-q32. Psychiatr Genet. $1995 ; 5(3): 117-126$.

10. Mulle JG, McDonough JA, Chowdari KV, et al. Evidence for linkage to chromosome $13 \mathrm{q} 32$ in a independent sample of schizophrenia families. Mol Psychiatry. 2005;10(5):429-431.

11. Chumakov I, Blumenfeld M, Guerassimenko O, et al. Genetic and physiological data implicating the new human gene G72 and the gene for D-amino acid oxidase in schizophrenia. Proc Natl Acad Sci. 2002;99(21):13675-13680.

12. http://www.ncbi.nlm.nih.gov/omim

13. Lin $\mathrm{CH}$, Lane HY, Tsai GE. Glutamate signaling in the pathophysiology and therapy of schizophrenia. Pharmacol Biochem Behav. 2012;100(4):665-677.

14. Burnet PWJ, Eastwood SL, Bristow GC, et al. D-amino acid oxidase activity and expression are increased in schizophrenia. Mol Psychiatry. 2008;13(7):658-660.

15. Yang HC, Liu CM, Liu YL, et al. The DAO gene is associated with schizophrenia and interacts with other genes in the Taiwan Han Chinese population. PLoS One. 2013;8(3):e60099.

16. Schumacher J, Jamra RA, Freudenberg J, et al. Examination of G72 and D-amino-acid oxidase as genetic risk factors for schizophrenia and bipolar affective disorder. Mol Psychiatry. 2004;9(2):203-207.

17. Bass NJ, Datta SR, McQuillin A, et al. Evidence for the association of the DAO (G72) gene with schizophrenia and bipolar disorder but not for the association of the DAO gene with schizophrenia. Behav Brain Funct. $2009 ; 5: 28$. 
18. Sacchetti E, Scassellati C, Minelli A, et al. Schizophrenia susceptibility and NMDA-receptor mediated signalling: an association study involving 32 tagSNPs of DAO, DAOA, PPP3CC, and DTNBP1 genes. BMC Med Genet. 2013;14:33.

19. Chung S, Jung J, Chung HY, et al. No association between polymorphisms of DAO and DAOA genes and homicidal behaviors in Korean schizophrenia. Psychiatr Genet. 2007;17(15):313.
20. Verrall L, Burnet PW, Betts JF, et al. The neurobiology of D-amino acid oxidase and its involvement in schizophrenia. Mol Psychiatry. 2010;15(2):122-137. 